\section{$\S 24$. On the Basis of Statistical Theory of Strong Turbulence in Inhomogeneous Plasmas}

Itoh, S.-I., Mori, H. (RIAM, Kyushu Univ.), Itoh, $\mathrm{K}$.

A statistical model for plasma turbulence is examined [1] by employing a basis from the Mori method [2]. The Mori method provides a base for the existence of the separation of the memory function from fluctuating force in the nonlinear dynamical equations. An analytic model for the turbulent damping coefficient is derived by use of the Green's function. The model formula takes a non-Markovian form, representing the property of the timereversibility of the basic equation. A recurrence formula is derived. The continued fraction form is also derived. A formula of the eddy-damping rate is derived. A rigorous relation which is derived by the Mori method is discussed, and is compared to the modelling. This comparison explains a nature of approximation in the modelling.

The dynamical nonlinear equations of fluctuations are given in terms of Fourier components, Eq.(2) is rewritten as

$\partial \mathrm{f}_{k} / \partial t+\mathcal{L}_{k}^{(0)} \mathrm{f}_{k}=\mathcal{N}_{k} \equiv \sum_{k=p+q} \boldsymbol{M}_{k p q} \mathrm{f}_{p} \mathrm{f}_{q} \cdot$,

where $\mathcal{L}^{(0)}$ denotes the linear operator, $J$ denotes the fluctuating fields, $f^{\top}=(\phi, J, h)$, and $\mathfrak{N}(f)$ stands for the nonlinear terms. According to the Mori's method, the nonlinear term is deduced to the memory function $\Gamma_{k}(s)$, and the fluctuating force $\mathcal{R}_{k}(t)$ as

$\partial f_{k} / \partial t+\mathcal{L}_{k}^{(0)} f_{k}=i \Omega_{k} f_{k}+\mathcal{R}_{k}(t)-\int_{0}^{t} \mathrm{~d} s \Gamma_{k}(s) f_{k}(t-s)$,

where $\Omega_{k}$ is the nonlinear frequency shift and.

The projection operators $\mathcal{P}$ and $Q$ are introduced $(\mathcal{P}+Q=1) . \quad \mathcal{P}$ represent the projection

$\mathcal{P} Y=\left\langle Y(t) f^{\prime}(0)\right\rangle\left\langle f(0) f^{\dagger}(0)\right\rangle^{-1} f(0)$,

where

$\left\langle A_{l}(t) A_{m}^{\dagger}(0)\right\rangle \equiv \lim _{T \rightarrow \infty} \frac{1}{T} \int_{0}^{T} \mathrm{~d} s A_{l}(t+s) A_{m}^{\dagger}(s)$

By use of this projection operator, the memory function $\Gamma_{k}(t)$, the fluctuating force term $\mathcal{R}_{k}(t)$ and nonlinear frequency shift $\Omega_{k}$ are given as
$\Gamma_{k}(t)=\left\langle\mathcal{R}_{k}(t) \mathbb{R}_{k}^{\dagger}(0)\right\rangle\left\langle f_{k}(0) f_{k}^{\dagger}(0)\right\rangle^{-1}$,

$\mathcal{R}_{k}(t)=\exp (t Q \Lambda) Q \mathcal{N}_{k}$,

$i \Omega_{k}=\left\langle\dot{f}_{k} f_{k}^{\dagger}\right\rangle\left\langle f_{k} f_{k}^{\dagger}\right\rangle^{-1}$,

respectively. The fluctuating force is orthogonal, i.e., $\left\langle\mathcal{R}_{k}(t) \stackrel{f}{ }(0)\right\rangle=0$.

By use of the dressed test mode method, we have the continued fraction expression for the Laplace transform of the memory function as

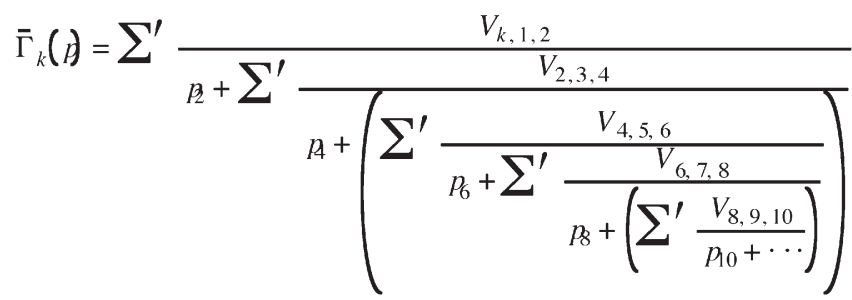

where

$$
V_{k, k_{1}, k_{2}} \equiv-2 M_{k, k_{1}, k_{2}} M_{k_{2}, k_{,-k}} C_{k_{1}}(s=0) \text {, }
$$

$p_{n}=p+\mathcal{L}_{k_{n}}^{(0)}$ and $\left\langle f_{k_{1}}(t) f_{-k_{1}}\left(t^{\prime}\right)\right\rangle=C_{k_{1}}\left(t-t^{\prime}\right)$ is the autocorrelation function of fluctuations. The eddy damping rate $\bar{\Gamma}_{0, k}$, at which the dressed test mode of $k_{j}$ decays due to turbulent damping, has a relation with the Laplace transform $\bar{\Gamma}_{k}(p)$ as $\bar{\Gamma}_{0, k}=\bar{\Gamma}_{k}(p=0)$, $\left(k_{j}=k, k_{1}, k_{2} \cdots\right)$. The expression for $\bar{\Gamma}_{0, k}$ by use of the continued fraction is deduced from Eq.(44). In the case of the matrix operator $\mathcal{L}_{k}^{(0)}$, the least-stable eigenvalue $\lambda_{k}$ is used, in the continued fraction as

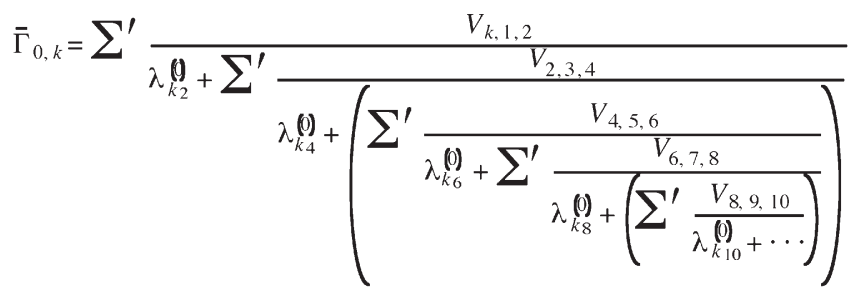

The approach in pervious work [3] is re-examined, and an analytic model for the turbulent damping coefficient is derived by use of the Green's function.

\section{References}

[1] S.-I. Itoh, K. Itoh, H. Mori: J. Phys. Soc. Jpn. Vol.75, No.3 (2006) 034501

[2] H. Mori: Prog. Theor. Phys. Vol. 33 (1965) 423

[3] S.-I. Itoh and K. Itoh: J. Phys. Soc. Jpn. 68 (1999)

1891, 2611; ibid. 69 (2000) 408, 427, 3253. 